\title{
Virtual endoscopic environments in modern neurosurgical practice
}

\section{Michael L. Levy, M.D., Joseph C. T. Chen, M.D., Ph.D., Arun P. Amar, M.D., Shinya Yamada, M.D., Ph.D., Koji Togo, M.D., Yoshiro lizuka, and Murwarid Mura Assifi}

Division of Neurosurgery, Children's Hospital of Los Angeles, Los Angeles, California; and Department of Neurological Surgery, University of Southern California School of Medicine, Los Angeles, California

Modern radiographic techniques have allowed the creation of high-definition planar images that can provide important anatomical as well as physiological data. Planar imaging sets can be reformatted into three-dimensional (3-D) data sets that can then be manipulated to demonstrate important anatomical or gross pathological features. Three-dimensional data sets have been used with success in modern image-guided or frameless stereotactic surgery. Another potential application is so-called "virtual endoscopy" or "scopeless endoscopy," in which a 3-D anatomical data set is reformatted into a volume-rendered image that can then be viewed. By reformatting images in this way, a "surgeon's-eye" view can be obtained, which can aid in presurgical planning and diagnosis. The use of virtual endoscopy has the potential to increase our understanding of the appropriate anatomy and the anatomical relationships most apparent during neurosurgical approaches. In so doing, virtual endoscopy may serve as an important means of planning for therapeutic interventions.

On the other hand, one must always be cognizant of the technical limitations of these studies regardless of the quality of the reconstructed images. Prospective, correlative, clinical studies in which the anatomical advantages of virtual-based endoscopy are evaluated in large cadaver or patient series must be performed. Until then, the only potential ways to compensate for errors that exist in the algorithms and reconstructions of 3-D endoscopic images are based on the surgeon's understanding of the clinical state of the patient and prior experience with the anatomy in the region of question.

Key Words * endoscopy * neuroendoscopy * computerized imaging * virtual imaging * virtual endoscopy

In recent years, there has been a growing tide of publications in which virtual techniques have been reported.[3,4,11,20,22,32,42-44] A number of factors have allowed for virtual endoscopic techniques to be introduced to the neurosurgical clinic and operating room. The most significant factor has been the general industry's utilization of virtual reality technologies. As mass production principles drive the cost 
of the requisite hardware down and refinements in design result in higher usability and power, many technologies initially conceived of for the general consumer market may find application in medicine. Another factor, the marked improvements in radiographic imaging, has resulted in higher-resolution images as well as new information modalities. This burgeoning of radiographic data presents new challenges and opportunities in the presentation and use of these data sets. Lastly, the introduction of endoscopy to surgical disciplines has given us a metaphorical basis (analogous to the desktop metaphor in personal computing) by which an interface can be created for data viewing.

Although the current published data on virtual endoscopy relate most significantly to imaging of the tracheobronchial tree[6,37] and gastrointestinal system,[4,48] detailed applications involving the central nervous system (CNS) have begun to be described.[3] This review represents an attempt not only to identify and explain the pathways by which virtual endoscopic interventions are completed but also the technologies currently available.

\section{VIRTUAL ENDOSCOPIC TECHNIQUES}

The techniques used in virtual endoscopy, like those in other technologically dependent fields, are likely to change profoundly in the coming years. Current limitations of virtual endoscopic techniques will be addressed as processor speeds increase and improved modifications of software algorithms, allowing for visualization of the appropriate anatomical structures, continue to be made.

There are five basic steps to the process of virtual endoscopy. These include radiographic data acquisition, data transfer, segmentation, rendering and viewing. We will review methods currently used in virtual endoscopy, placing particular emphasis on limitations of current technology. Thereafter, we will review specific available implementations of this technology.

\section{Radiographic Image Acquisition}

Computerized tomography (CT) and magnetic resonance (MR) imaging in general acquire two-dimensional (2-D) planar images from which 3-D images can be generated using appropriate software. The quality of the eventual 3-D reconstruction depends on the mode and resolution of image acquisition. Both slice thickness and image matrix size are basic determinants of final resolution.

In soft-tissue imaging of the head, neck, and spine, MR imaging has been and likely will continue to be the imaging modality of choice. Continuing refinements in MR imaging sequence protocols, field strength, and gradient coil design have been important in the improvement of final image quality.

Although CT scanning in general cannot provide the same spatial detail as found on MR imaging, significant progress has been made that ensures its continued usefulness in head, body, spine, and bony imaging. An important advance in CT scanning has been the introduction of helical or spiral scanning. Whereas conventional axial CT scanning involves progressive table movements with exposure to $\mathrm{x}$-radiation, spiral CT scanning involves the simultaneous table movement in conjunction with $\mathrm{x}$-ray exposure.[21] The x-ray path consists of a helix or spiral curve around the patient. Helical CT scanners can reduce acquisition time, thereby reducing motion artifacts. Other advantages of spiral CT specifically related to virtual endoscopy are the minimization of respiratory and motion artifacts and its ability to provide overlapping data image sets without exposing the patient to additional radiation. Spiral CT produces a single continuous data set that represents a volume of tissue. Contiguous data sets are required without interruption between slices to create the most appropriate 3-D reconstructions. The operator can then determine the varying degrees of image overlap, and a 3-D image is generated that 
allows for the optimization of image quality. Obviously the higher the resolution of the initial data set, the more appropriate and anatomically correct is the 3-D reconstruction. The operator can include thinner slices, which are obtained over a smaller area of interest, allowing for the maximization of spatial resolution. If possible, tissue contrast can be maximized by the operator to assist the computer further in differentiating the salient anatomy.

Spiral CT scanning has been the preferred means of obtaining images in systemic organ systems such as the gut, because the rapid acquisition times reduce movement artifact generated by peristaltic and respiratory movements. Although it provides less anatomical detail than MR imaging, it remains an important imaging modality for virtual neurendoscopy. Optimum scanning parameters for each procedure have not yet been identified in spiral CT-based virtual endoscopy. Obviously the maximum resolution of any structure will be obtained using the smallest beam collimation possible. This, in conjunction with the slowest table speed and maximum degree of reconstruction-slice overlap, should further enhance the detail of the image. In addition, kilovoltage and milliamperage should be maximized to improve signal to noise ratio. The unfortunate correlate of the aforementioned is that the optimization of such scanner parameters can result in increased radiation exposure. A middle ground must be reached between these optimum scanning parameters and the reality of visualization in light of the surgical requirements for anatomical detail and the need to avoid exposing the patient to excessive radiation. In a recent study in which virtual colonoscopy and conventional colonoscopy were compared, the radiation dose in patients was considered to be low and was calculated at approximately $0.0044 \mathrm{~Sv}(0.44 \mathrm{rem})$ per scan. This was equivalent to the radiation exposure that results from obtaining two abdominal radiographs.[21] Obviously, significant efforts will be required to create software/hardware that can be used to address this problem.

In a recent study typical helical CT data acquisition parameters were designated for postprocessing 3-D reconstructions during virtual colonoscopy.[20] These parameters were as follows: $120 \mathrm{KVP}, 165$ milliamps, 20 to 40-second exposures with 5 to 3 -mm collimations, and 5 to $6-\mathrm{mm} / \mathrm{second}$ table feed (pitch 1:2). The matrix was 512 X 512. Reconstruction parameters included 180-linear interpolation standard reconstruction kernels and 3-mm table incrementation.

Virtual endoscopic examinations of the tracheal bronchial tree have further elucidated the necessary quality of scan parameters required during image acquisition.[37] In one study endoluminal space-occupying lesions of greater than $5 \mathrm{~mm}$ and stenosis of greater than $50 \%$ were detected quickly by virtual endoscopy in all cases. In addition, altered configurations based on external compression were always correctly identified. Superficial spreading tumors and lesions smaller than $5 \mathrm{~mm}$ were not detected during imaging. A pitch of less than $1 \mathrm{~mm}$ is usually not adequate, given the proportional increased radiation exposure required, as we previously discussed. For collimations of 7 to $10 \mathrm{~mm}$, partial volume-averaging artifacts can also render the quality of the axial slices and 3-D endoscopic images unsuitable for diagnostic purposes. It has been concluded that the upper limit for collimation is either $5 \mathrm{~mm}$ with a pitch of 1 or $3 \mathrm{~mm}$ with an increased pitch of $2 \mathrm{~mm}$.[37] For practicality, it was determined that a collimation of $3 \mathrm{~mm}$ with a pitch of $1.5 \mathrm{~mm}$ and an overlapping reconstruction with an increment of $1 \mathrm{~mm}$ is optimum. These parameters maximize the data for producing 3-D reconstruction while minimizing the amount of radiation used in obtaining the study.

True axial images from MR imaging[9,39] or nonhelical axial CT data sets can also be reconstructed into 3 -D models, but this requires longer acquisition times that can result in further loss of anatomical detail. 
For most neuroendoscopic studies, MR imaging remains the modality of choice. Because the structures of the head are relatively immobile, extended acquisition times are possible and thereby allow for generation of high-resolution images. Over the last 15 years, MR technology has improved greatly. Improvements in field strength, sequence architecture, and gradient-coil technology have resulted in an ability to obtain higher-resolution images in shorter acquisition times. Experimental high-field-strength-magnets currently being used on an experimental basis have generated images of breathtaking clarity. The amount of data generated by such systems provides ideal raw material for use in virtual endoscopic applications.

\section{Data Transfer}

Following initial imaging, the data set must be transferred. This is most often accomplished via a network connection but also through DAT tape, optical disc storage transfer, or CD-ROM transfer. Historically, transfer of data sets for 3-D processing has been difficult due to the proprietary image formats of individual systems. Reverse engineering of these data formats was required by experienced computer software engineers. Because of problems regarding proprietary data formats, advancements in radiographic image manipulation were held back, lagging far behind other disciplines in which image manipulation is commonly employed, such as in multimedia or desktop publishing. The radiographic technology industry, recognizing the negative effects of lacking standard measures on the development of the industry, has begun to move toward standards-based formats. An example is the creation of the Digital Imaging and Communications in Medicine standard, which is currently being widely adopted, having been endorsed by most major vendors.

\section{Segmentation Process}

For the cross-section image data sets obtained from CT or MR imaging to be reconstructed into a 3-D anatomical surface or volume, segmentation of the data points needs to be achieved. "Segmentation" refers to the process by which neighboring anatomical features are differentiated. Each voxel is initially classified based on its signal density. Once all initial representative voxels are classified into bone, air, fluid, or tissue (initial seeds) any connected voxels are marked with similar unique identifiers. A critical step in actually defining the salient anatomical correlates for the endoscopic viewing is to establish the data thresholds. All voxels containing tissue of a certain range of CT or MR imaging attenuation are given a specific label. This process has been typically accomplished by painstaking manual techniques, although a number of automated methods are being developed.

A variety of approaches has been attempted to achieve automated segmentation[8] including techniques dependent on simple thresholding,[17] edge extraction,[24] clustering algorithms,[35] elastic matching,[47] fuzzy logic,[30] knowledge bases,[7] region growing,[18] and pattern recognition.[41] Clearly, the multiplicity of approaches devised is a byproduct of the intense research into the field of artificial intelligence.

Regardless of the specifics of the technique, several fundamental principles are common to all segmentation methods. First, establishing the proper thresholds for the raw data set must be accomplished to create accurate boundaries that will be extracted by the segmentation method. Images that are oversaturated may tend to overstate the boundaries of certain anatomical features, whereas undersaturation may result in loss of definition of fine structures. Second, most modern segmentation methods take advantage, to some extent, of generalized maps of "normal" anatomy to serve as a guide to the segmentation algorithm. Third, in an increasing number of MR-based segmentation algorithms, 
multispectral information is processed to increase the likelihood of differentiating neighboring voxels. The development of automated segmentation techniques is crucial to the development of virtual endoscopic methods. Advancements directed at improvements in radiographic image quality as well as improved segmentation algorithms will be required.

\section{Rendering Methods}

Pixels are the points that comprise 2-D sets of visual data, such as that displayed on a computer screen. Voxels (volume pixels), on the other hand, are the 3-D correlates of pixels and are the smallest volumetric pieces of information that compose 3-D imaging sets such as CT or MR image. Each voxel in an MR or CT study has a defined $\mathrm{x}, \mathrm{y}$, and $\mathrm{z}$ dimension. "Rendering" is the process by which a 3-D data set consisting of voxels is reconstructed into an image representative of a physical form with realistic lighting and shading. In the rendering process, each voxel is assigned a color or opacity based on the operator's use of different algorithms. In addition, a perspective viewpoint is assigned during the rendering process. Rendering algorithms can determine the reflective nature of the surface, lighting direction, and lighting coordinates, and the actual surface appearance can be manipulated and rendered by the operator to give it a more realistic appearance. Various approaches have been undertaken in the rendering process. Specific techniques include ray tracing, radiance, and parallel and scanline algorithms. Although an indepth discussion of the multitude of rendering techniques is beyond the scope of this paper, some general definitions and principles are worth exposition.

The rendering process can be divided into two separate phases: geometry and shading. "Geometry" refers to the creation of a perspective viewpoint within 3-D space. "Shading" refers to the process by which lighting, shadows, and texture are added to the image. Ray tracing is the classic method of rendering used in the creation of high-definition computerized effects and animation. In this method, a virtual light source is defined. In the case of virtual endoscopy, the light source is arbitrarily defined to be coincident with the viewpoint. A mathematical model is then constructed of light rays as they emanate from the light source and bounce around the virtual world. The method allows for the creation of highly realistic images that are complete with appropriate texturing, reflections, and shadows. The drawback to this method is the amount of computational power, and therefore time, required to render a single image. Thus, it is not used in real-time interactive virtual reality-type displays.[15]

In most interactive virtual reality-type display systems, as exemplified by modern interactive game systems (RAVE, Direct 3D and OpenGL), a combination of specialized hardware and software is used to render images. Rather than using ray tracing techniques, a technique known as "scanline" rendering approximates results that one would obtain from ray tracing with far less processor overhead. Although scanline images tend to result in less realistic images, they can be generated in real-time fashion. In most images rendered by commercially available medical packages use some implementation of scanline rendering is used.

Surface Compared With Volume Reconstruction. Most images generated by rendering techniques are of opaque objects. Translucent or transparent objects, however, may also be rendered, albeit with a higher processor overhead. Opaque-object rendering has been termed a "surface reconstruction" and translucent/transparent-object rendering has been termed "volume reconstruction." Both surface- and volume-rendered techniques have been used in virtual endoscopic displays.[39,40,44,46]

Surface-rendered techniques allow one to analyze information acquired from the surface of a data set. Volume-rendered techniques utilize the complete 3-D data set and allow for the display of the 
reconstructed inner surface as well as the outer surface.[20] The latter is more costly when one considers disc storage and the calculation time required for the regeneration of the 3-D reconstruction.

Image Manipulation and Viewing Techniques and Interfaces. Having created a 3-D data set, a crucial determinant of usability is the interface. Three techniques have been described to guide virtual cameras through reconstruction models. The first is a manual-based modality in which a mouse is used to change the camera position and focal point. The camera is maintained within the structure of interest, whether it is the cerebral ventricle or subarachnoid space by providing collision detection of the visual focal point with the anatomical surface. This allows for the focal point to remain within the cavity in question.

A second modality is described as key framing, an automatic procedure in which two selected points, as identified by the operator, are interpolated and then passed by the software algorithm. Cubic splines are used to calculate intermediate parameter values at the resolution of the operator's choice prior to movement in the environment.[20,23] The key camera location and orientation are manually controlled. The final virtual camera model is that of automatic path planning. Such planning is based on the difficulty in traveling through hollow structures of some complexity. The goal of the exploration is to find the terminus of the organ that is being explored, and then the operator generates a starting point. The computer determines the shortest path between the origin and end point staying within the constraints of the organ cavity as defined by its walls, which are considered the obstacles. The shortest path is determined using a steepest-descent algorithm protocol. The path can subsequently be smoothed prior to display.

An emerging alternative to such planning is the integration of a haptic (force-feedback) workstation connected to the control mouse, which allows one to stay within the subarachnoid space or cerebral ventricles. This ensures that when the focal point reaches the surface plane, differentiating between the interior and surrounding exterior of the hollow organ of question, force feedback into the joystick will prevent movement outside of the interior. We have evaluated force-feedback or haptic workstations in efforts to facilitate virtual endoscopic movement within the interior of the ventricular system.[16] With regard to automatic path planning, the camera is considered to be a point and the organ walls considered to be obstacles, which can be identified in 3-D space.[26]

Presentation of virtual endoscopic images also provides challenges. In most implementations, three windows are simultaneously displayed on the monitor, including the global 3-D view, local views, and the correct CT or MR axial, sagittal, or coronal slice that most appropriately documents the placement of the endoscopic tip. The surface-rendered perspective and the virtual endoscopic internal view can be displayed from various angles simultaneously. Single-image display represents traditional surface rendering of an organ, which can also allow for transparent renderings of enclosing tissues such as skin or bone.

It is desirable, however, that 3-D data sets be displayed in a 3-D fashion, that is, stereoscopically. Various implementations for stereoscopic display are currently available. Stereoscopic images can be generated using software algorithms with concurrent visualization using liquid crystal display shutter glasses (3-D TV Systems, San Raphael, CA), high-resolution workstations with liquid crystal display shutter glasses (Stereographics, San Raphael, CA), large-screen projection in which polarized glasses (VREX, Hawthorne, NY) are used or via stereo head-mounted display systems (Vista Medical, Carlsbad, CA).[28] A final display technique is that of split-screen display in which an image obtained from a user-specified location that allows one to localize the tip of the endoscope stereotactically is present 
concurrently with the simulated endoscopic view.[29] This display allows one to localize the virtual endoscopic "tip" at any time in 3-D space via a pointer-display command key.

\section{SPECIFIC IMPLEMENTATIONS OF VIRTUAL ENDOSCOPIC SYSTEMS}

Any discussion of specific implementations of virtual endoscopic systems must be tempered by the recognition that computer technology continues to advance rapidly. Gordon Moore, a founder of the Intel Corportation, observed in 1964 that available computer processing power tended to double every 2 years. Remarkably, this observation has held true over the last 25 years, with no clear evidence of deviation. Because of the ephemeral nature of computing technology, it is likely that the specifications of these systems will change drastically over the next few months and years. With the wider availability and greater power of computer systems, costs will also decrease significantly. It is unlikely, therefore, that current implementation of virtual endoscopy will continue to be used 3 years from now. Despite this fact, it is useful to examine specific representative implementations to understand how various vendors have attempted to solve problems associated with this technique.

A multitude of systems are currently available for virtual endoscopic rendering.[1,3,4,22,38,49], and most utilize proprietary software associated with specific helical CT scanners such as Picker (Picker PQ 5000-CT Spiral CT Scanner and the Episcope software package; Picker International, Inc., Cleveland, $\mathrm{OH})$, General Electric ([GE] Pro-Speed Advantage Helical Scanners and Advantage Navigator software; GE Medical Systems, Schenectady, NY), and Siemens (Siemens Slip-Ring Spiral CT, Iselin, NJ). These software packages can also accept data from respective MR devices.

\section{General Electric Products}

Advantage Navigator is a software package used for virtual endoscopic reconstructions of datasets that are imaged on GE Pro-Speed Advantage helical CT scanners and Signa MR imaging systems. Techniques for the use of helical CT scanning have been described for the acquisition of anatomical volumes used specifically to evaluate facial bones, the skull base, and the perinasal sinuses.[10] The technique described for these studies includes 3-mm slice thickness with a table speed of $3 \mathrm{~mm}$ per rotation (pitch 1:1). Rotation times for the tube are appropriately 1.5 seconds for $360^{\circ}$ with acquisition parameters of 120 kilowatts and 160 milliamps. The acquired slice is then reformatted at an interval of $1.5 \mathrm{~mm}$ by using a detail filter. Auer and Auer have taken advantage of MR-based imaging when using $\mathrm{T}_{1}$ - weighted echo gradient images that were acquired at a resolution of $0.9 \times 0.9 \times 1.0-\mathrm{mm}$ voxels. Once the images are acquired, the data are transferred to a GE Advantage Windows workstation based on Sparc 20 platform (Sun Microsystems, Mountain View, CA) where they are postprocessed. The high-resolution slices can then be reformatted to generate a 3-D surface image, with other slices being maintained in the coronal, sagittal, or axial plains. The Navigator software allows for reconstruction of endoscopic images by using either high- (512 X 512) or low-resolution ( 256 X 256) matrices. The operator can assign color to tissue. Subsequent fly-through techniques can be created within the lumen or hollow surface area by creating a flight path while the camera is positioned along the endoscopic path at the tip of the endoscope. The surgeon can also define the position of the camera along the endoscopic path based on the current location of the camera and the appropriate direction, which is established by formulating a number of checkpoints.

Jolesz and associates[20] have used a Lymb programming and rendering environment to reconstruct the virtual images (GE Medical Systems). Most of their work was completed on a SUN workstation equipped with a ZX graphics accelerator board. They used the $\mathrm{C}$ programming language and 
Unix/X-windows operating environment to create their reconstructions. Their virtual endoscopic software allowed for translation and rotation around a viewpoint that one would obtain with a normal endoscope. Movements included advancement along the Z-axis, rotational deviation around the Z-axis, and pivoting around the $\mathrm{X}$-axis. The actual simulation was completed on a Silicon Graphics Indy workstation with a MIPS R 4400 processor chip (Silicon Graphics, Mountain View, CA).[20]

In one study documented the use of virtual endoscopy based on CT angiography in a patient with a basilar artery aneurysm was documented.[31] All studies were performed by using a GE High-Speed Advantage CT Scanner (GE, Milwaukee, WI) with the helical acquisition using $140 \mathrm{ml}$ of omnipaque 300 at a rate of $2.5 \mathrm{ml} / \mathrm{minute}$ via a power injector. Acquisitions started at 25 seconds after the beginning of the contrast injection, with a table speed of $1 \mathrm{ml} / \mathrm{second}$ and 1-mm collimations with a 1-to- 1 pitch and a $15-\mathrm{mm}$ field of view (FOV). The matrix was established at 512 X 512. The data were processed using an Advantage Windows workstation (GE), first by using a 3-D maximum-intensity projection reconstruction (Voxtool Software). Despite the quality of the image, the maximum-intensity projection analysis failed to determine the exact origin of the aneurysm and its relationship to the adjacent vessels. By using the Navigator software, their reconstruction was performed with a surface display algorithm at a lower threshold of 871 Hounsfield units. The virtual endoscope was set on a reformatted coronal view a few millimeters from the origin of the left posterior cerebral artery and oriented toward the basilar artery. The aperture angle was $60^{\circ}$. With these changes the endoscopic tool confirmed that the 3-mm aneurysm lacked a neck at its origin and partly involved the right anterior superior cerebellar artery.

\section{Siemens Products}

In another series evaluating virtual endoscopic approaches to the paranasal sinuses the authors used data that were obtained on a Slip-Ring Spiral CT (Siemens Medical Systems, Iselin, NJ).[14] The imaging data were transferred to an Onyx workstation by utilizing the Reality Engine II Graphics package (Silicon Graphics).[14] Image processing was performed with a VoxelView software version 2.1.2 (Vital Images, Fairfield, IA). Noncontributory data was disposed of using a technique termed "histogram truncation and compression," thereby conserving disc space. The decision to truncate was determined by the operator for the particular anatomical study. Truncation techniques allow for visualization of information that is believed to be appropriate by the operator and is defined for the specific anatomical reconstruction. If included in the reconstruction, the discarded information would only needlessly slow down information processing and manipulation. An additional methodology of performing a fly-through by finding key locations was also described by Gilani, et. al. Because each of these key locations was then connected automatically by the computer, continuous fly-through or visual walk-through was permitted. The spatial coordinates defining viewer's placement in space and the viewer's direction of view from that location in space, in addition to the FOV at each key point, was included and described by the operator. The flight path was then designed. For most flight paths approximately 150 points can be chosen that will result in approximately a 5-minute fly-through time. The FOV is based on the degree of endoscopic angulation (that is, $0,20,45$, or $90^{\circ}$ ) that the operator determines is most appropriate should an actual endoscopic intervention be undertaken. This interconnecting path of key points is also known as a "spline path" and interconnects the key images through the shortest line path between each of the key frames. The spline curve can be generated at a rate of 30 frames/second if there are discrepant imaging findings on key images as defined by the operator. The program will automatically smooth the path between the different key points by using a specific algorithm. Visualization can then take place via monitor or by transferring the data onto videotape for later viewing. 
In a recent study 3-D MR angiography was evaluated in the planning of aneurysm surgery in a series of 20 patients, and a 1.5 tesla Siemens Magnetron System was used.[34] For arterial flow the presaturation band of the MR angiographic image was placed above the region of interest, which allowed for elimination of venous flow from the region. The authors then created a maximal-intensity projection from the original MR angiography data, which isolated the vessels of interest and suppressed the signal of the surrounding structures. The authors used two high-resolution MR angiography sequences based on commercially available 3 -D gradient echo fast-imaging steady-state precision sequences. The acquisition parameters used were repetition time at $44 \mathrm{msec}$, flip angle of $20 \%$ with an acquisition matrix of $256 \mathrm{X}$ 256 in an FOV of $230 \mathrm{~mm}$. The 3-D slabs were of $90-\mathrm{mm}$ thickness partitioned into 64 slices with an acquisition time of 8 minutes. The total imaging time for each study was approximately 20 minutes. The data set was then transferred to a multi-vendor protocol computer by a PAC-Net network protocol by utilizing a TCP/IP network protocol. The reconstruction was then created using a neurological surgery planning system, version 3.0, on a Sun SPARC 10 workstation (model-514 Sun Microsystems Computer Corporation).[49]

In another report, the system utilized the Somatom Plus 4A Scanner (Siemens, Erling, Germany).[25] Images were obtained based on the collimations and reconstructions as determined by the surgeon. Images were then magnified using the protocol of Kernel AB10. Three-dimensional images were obtained using a shaded surface display technique with the Magic View version VA02C. The images were regenerated and reconstructed using the shaded surface display with a threshold determined by the surgeon.[25]

\section{Ideas Med-Link Software}

We currently use software that allows for a mathematical representation of all data within the 3-D volume.[16,27] The Ideas Med-link Software (Dynamic Computer Resources, Inc., San Dimas, CA) uses splines to make up the surface of the rendered dataset. Because splines have control points they can be adjusted using a workstation either via a mouse, joystick combination, track ball, or haptic (force-feedback) workstation. Such manipulation can allow for the reconstruction of craniofacial or other virtual image sets that permit virtual surgical reconstructions and reformulation of abnormalities involving the CNS. Such software allows the interaction and manipulation of the data set which cannot be achieved with view-only software.

\section{Potential Advantages of Virtual Endoscopy}

Endoscopy is an optical, video-assisted technology that allows the operator to change the position of the endoscope to facilitate views as well as visual information generated during an intervention or diagnostic procedure. It is likely that the resolution of virtual endoscopic 3-D reconstructions based on CT or MR imaging data sets will never achieve that of true optical endoscopes. In addition, software-based integration of this cross section of data into 3-D images may not be anatomically accurate based on the rendering algorithms used. Despite this, the use of virtual endoscopy can provide important information to the surgeon. Such information includes data regarding tumors that may extend through or into fluid filled spaces such as the ventricular cavities.[2,3] This information allows not only for the localization of the lesion in question but also better defines its relationship to adjacent anatomical structures.

Future hardware and software based developments in virtual techniques, as well as the incorporation of stereoscopic visualization, will likely enhance the quality of image representation. In addition, display methodologies can be designed to mimic specific diagnostic or surgical approaches. As previously noted, 
these can be similar to endoscopic views with split screen or picture-in-picture renderings of 3-D localization during the procedure. Furthermore, the incorporation of stereoscopic visualization, which can be simply created using algorithms and pixel redistribution, can generate more realistic images.

It is likely that virtual endoscopy will aid exploration into both the ventricular and subarachnoid spaces now and in the near future. Obviously, such interactions can depend completely on the surgeon without limitations based on surrounding anatomical structures. Ventricular and subarachnoid space fly-throughs can be performed using surgeon-controlled or automated techniques. In manual control, the visualization can be completely interactive with surgeon controlling of all camera parameters and FOV. Infinite depths of field and the fact that the virtual presentation can pass out of fluid-filled cavities into solid structures are further benefits. In addition, given the 3-D positioning of the specific view, be it endoscopic or otherwise, it can always be accurately localized and integrated with frameless stereotactic navigational systems. This is in addition to the use of endoscopy concurrently with real-time imaging guidance or interventional MR imaging.[12,13,19] Another benefit given the volumetric nature of the data set, is that one can measure the size of an anatomical abnormality, tumor, or vascular malformation as well as information regarding depth or volume in any arbitrary orientation. This may guide subsequent endoscopic or surgical approaches. We would recommend concurrent imaging of a sphere of known volume during the actual imaging procedure to verify the accuracy of the volume that was determined by the CT or MR imaging.

Virtual endoscopy allows for an interactive operator-determined exploration within the inner or surrounding space of an organ system. Concurrent information regarding the relationship of the viewpoint to anatomical structures either located within or outside of the hollow structure can be displayed (Case 1, Figs. 1-11).[20] The use of 3- or 2-D pointers can also be used simultaneously to identify the location throughout the fly-through. The demarcation of contrasting margins, as when MR angiography is performed in cerebral vasculature, cerebrospinal fluid-filled spaces of the subarachnoid, or ventricular cavities or areas of enhancement following contrast administration, allows for the accurate delineation of these structures, and 3-D reconstructions can be quite quick and fully automated.

Case 1: Three-dimensional virtual endoscopic reconstruction of ventricular cavity and periventricular region. Concurrent injection of Iohexol intraventricular contrast via a ventricular catheter and intravenous Ioversol (for 3-D cerebral angiography) sequential data were obtained using 2-mm-thick slices with no gap. Voxel transmission calculations utilizing 1-mm slices were then completed to reconstruct and correlate the ventricular and surrounding vascular anatomy. The voxel method was then used for obtaining virtual endoscopic images (surface images) of the ventricular chambers. 


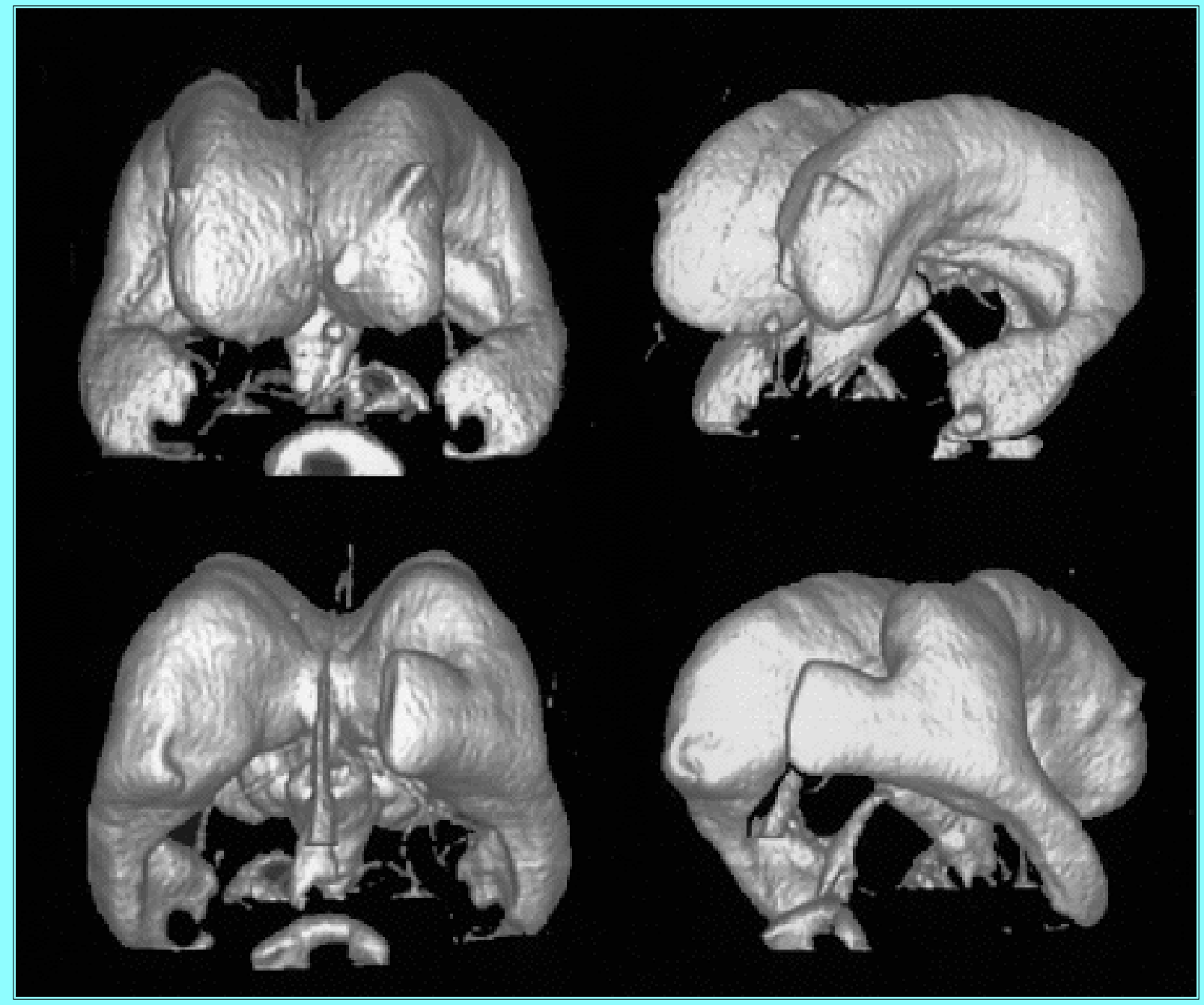

Fig. 1. Initial virtual reconstruction of ventricular chamber (surface reconstruction) by using the voxel transmission method. With rotation, all aspects of the external ventricular anatomy can be visualized. 


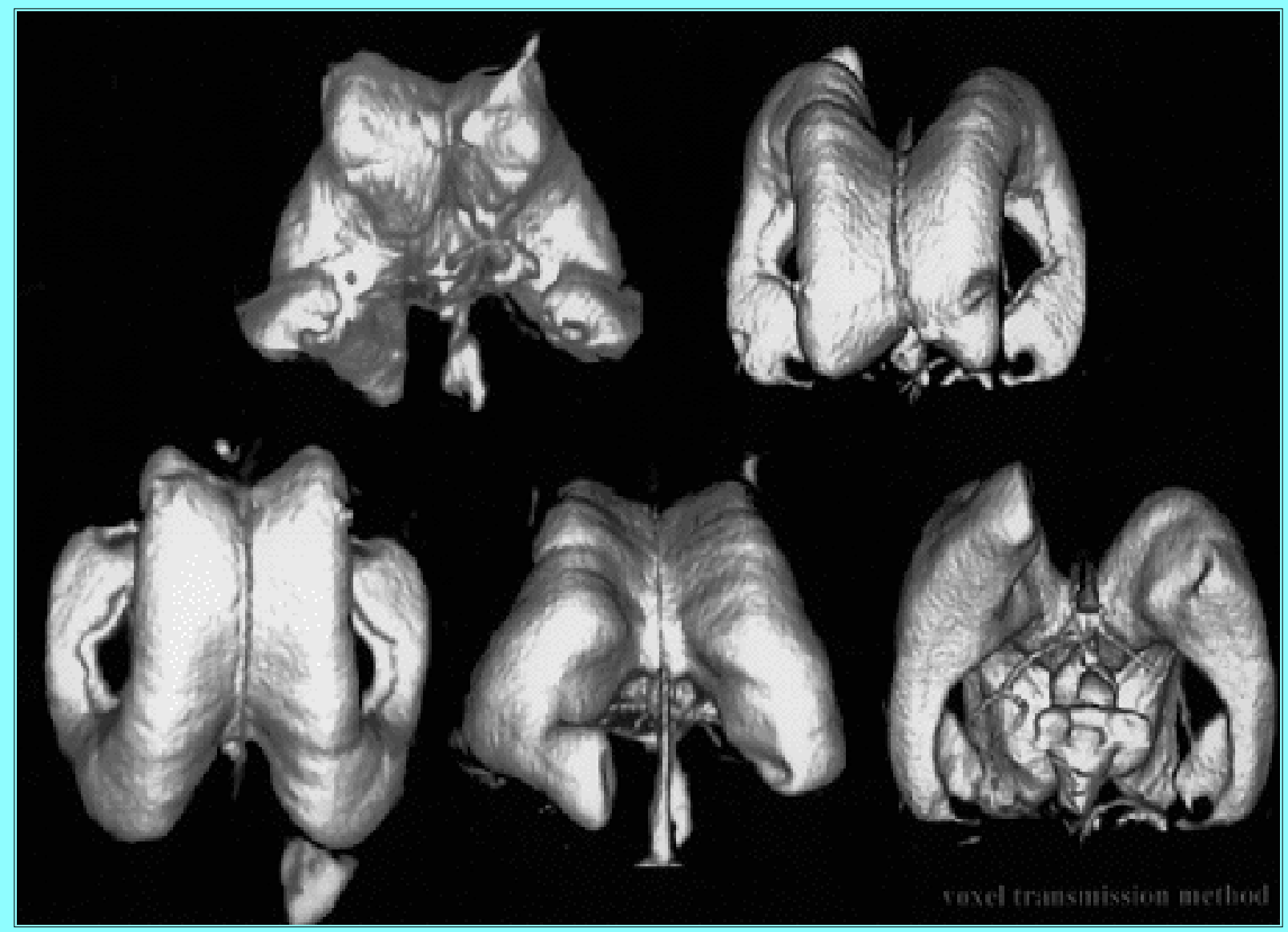

Fig. 2. Virtual reconstruction of ventricular chamber (surface reconstruction) in which the voxel transmission method was used (superior, posterior, and anterior views).

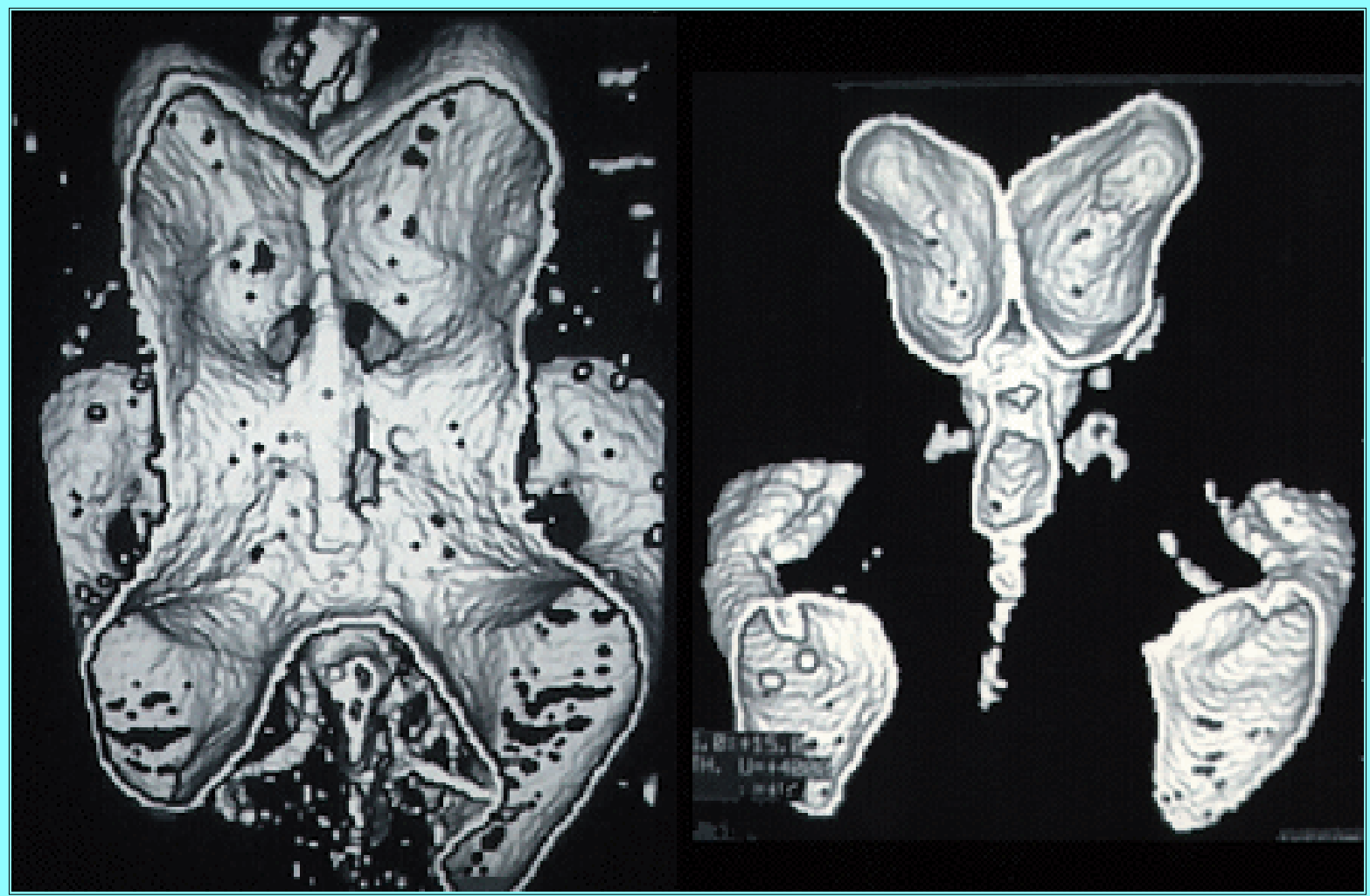

Fig. 3. Reconstructed virtual endoscopic view of the inner surface of the lateral ventricular floor. 


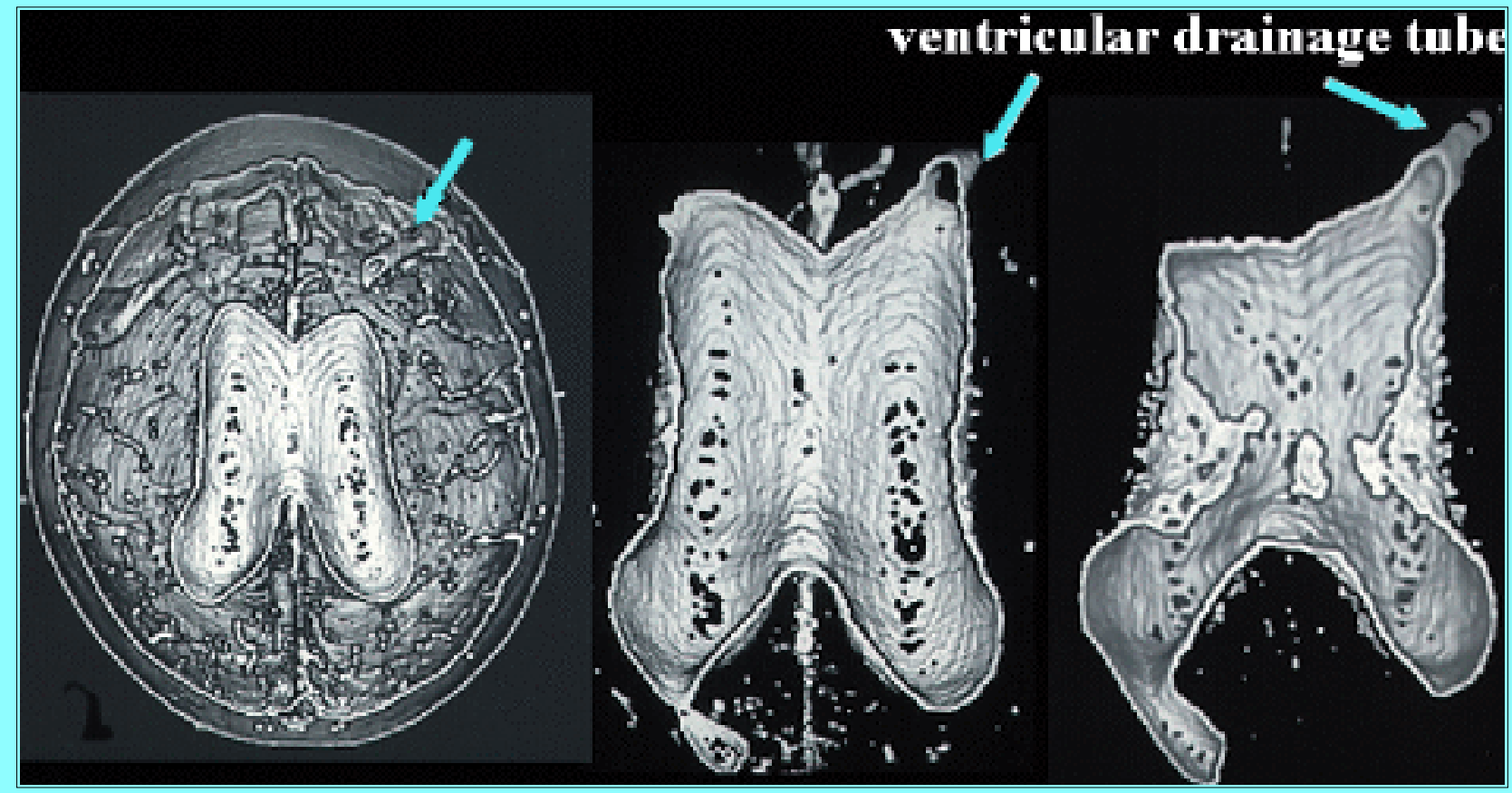

Fig. 4. Reconstructed virtual endoscopic view of the inner surface of the lateral ventricular roof. The entrance of the ventricular catheter drainage tube is also demonstrated (arrow) in each image.

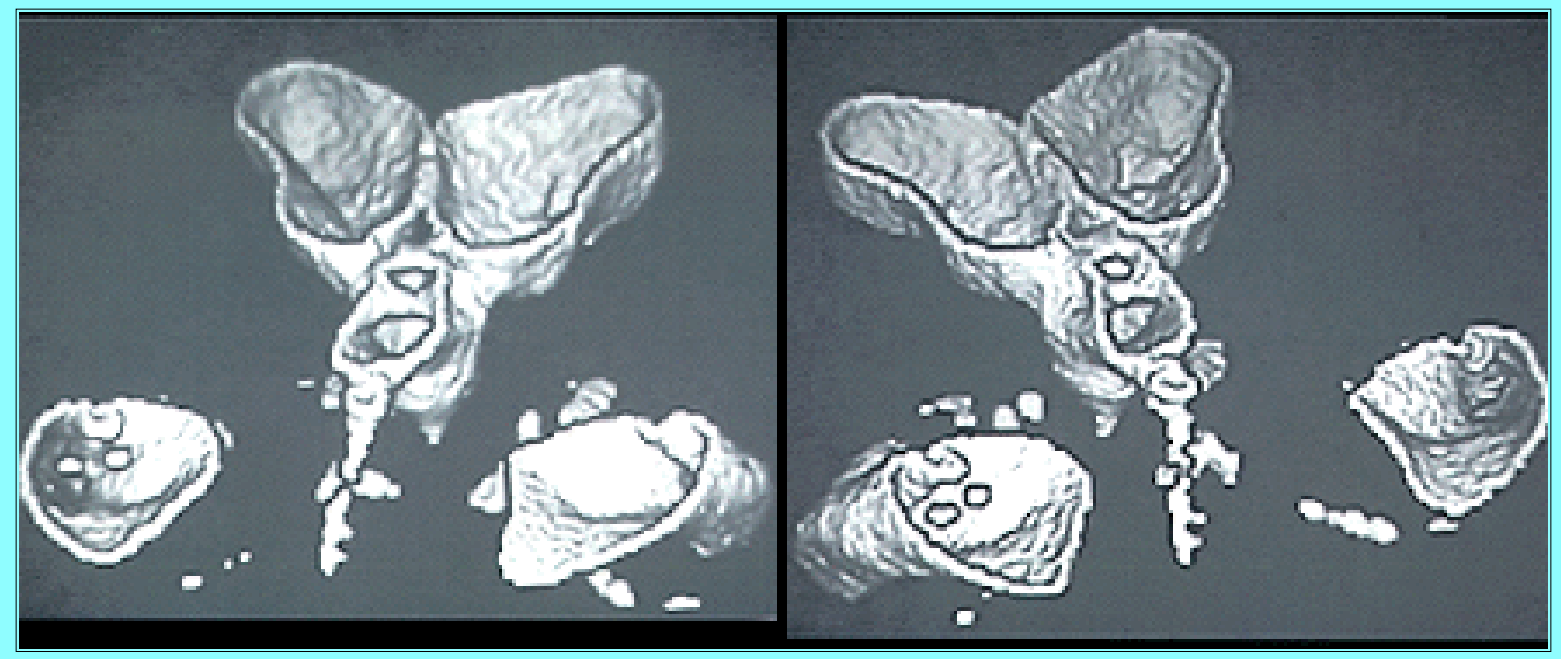

Fig. 5. Reconstructed virtual endoscopic view of the inner surface of the inferior and anterior horns of the lateral ventricles. 


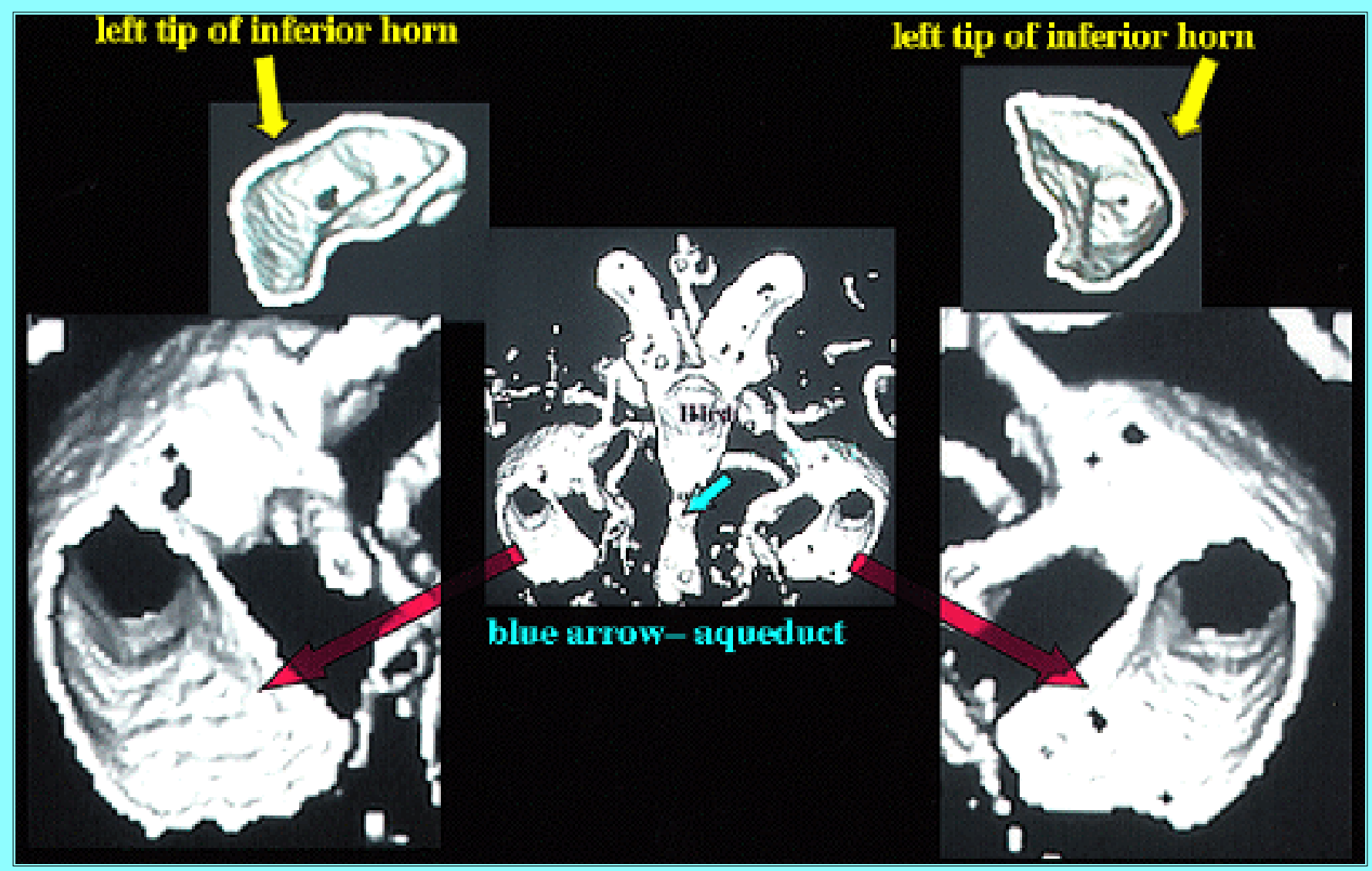

Fig. 6. Reconstructed virtual endoscopic view of the inferior horns of the lateral ventricular floor. Note the view of the third ventricular chamber and aqueduct. 


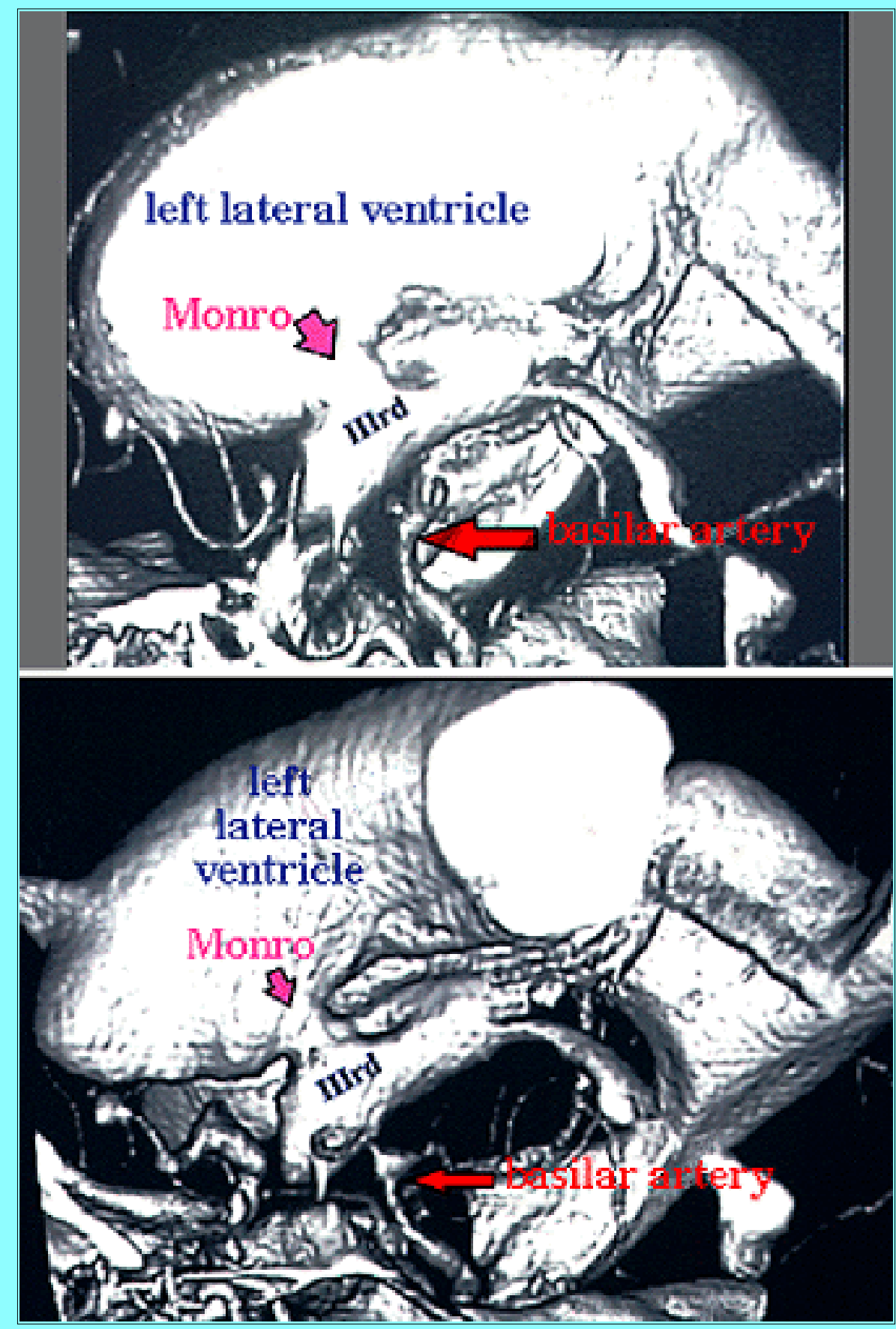

Fig. 7. Voxel transmission reconstruction of the anatomical relationships between the basilar artery and floor of the third ventricle (IIIrd). 


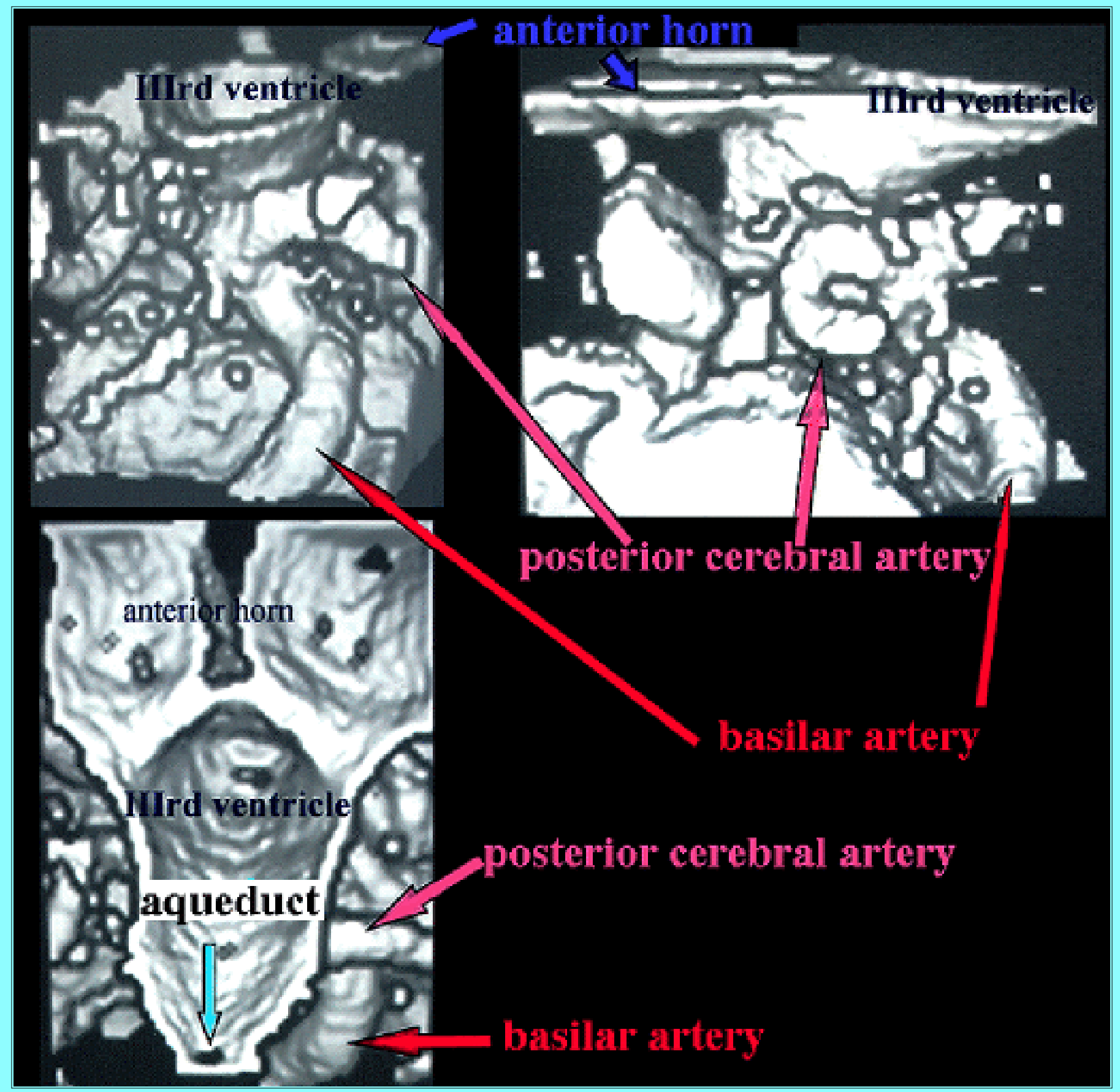

Fig. 8. Reconstructed virtual endoscopic view of the third ventricle depicting the relationship of the posterior cerebral artery and basilar artery. 


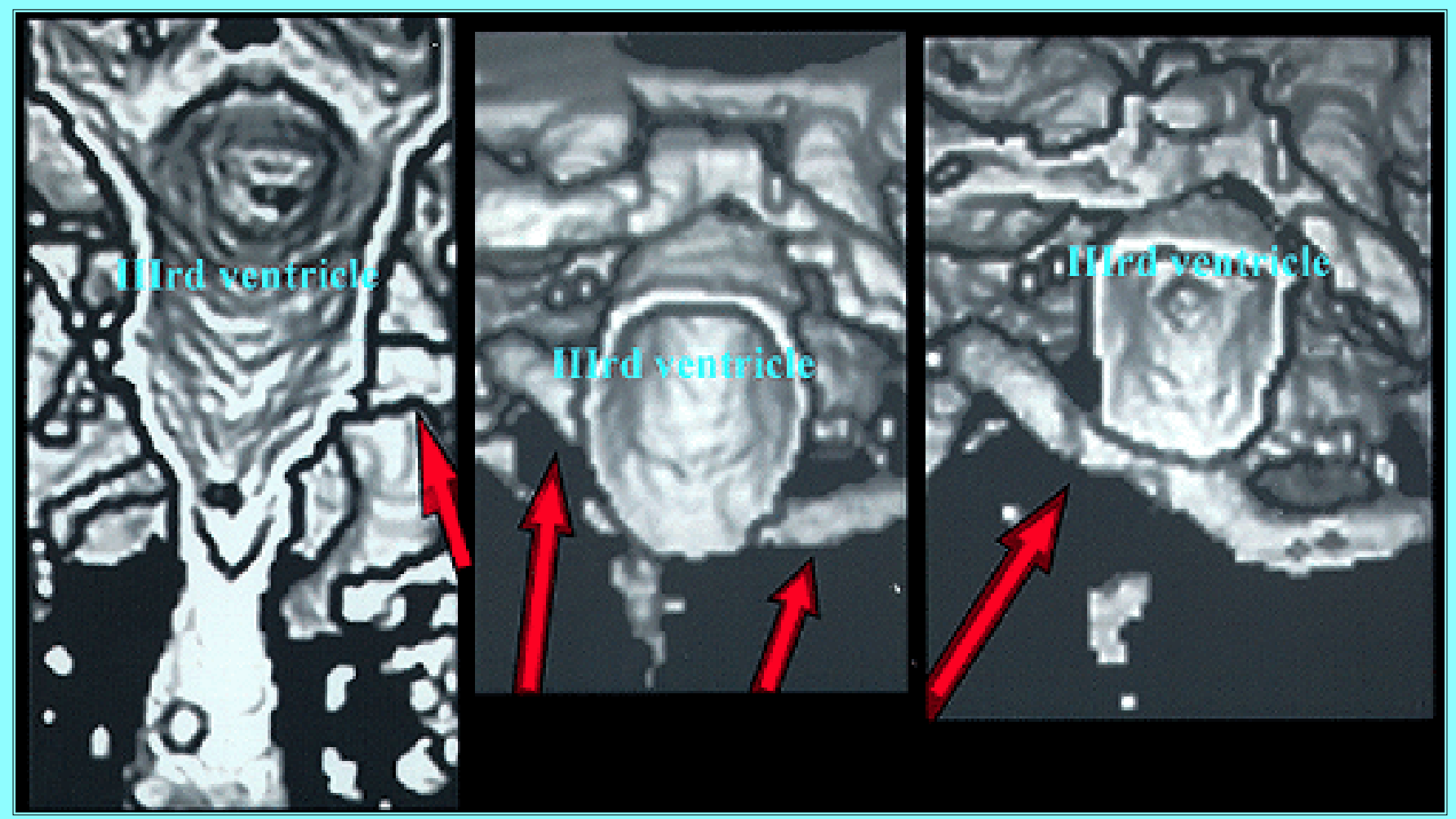

Fig. 9. Reconstructed combination of a virtual endoscopic view of the third ventricle and 3-D CT cerebral angiography showing accurate anatomical positioning of the posterior cerebral artery (arrows) to the ventricular floor.
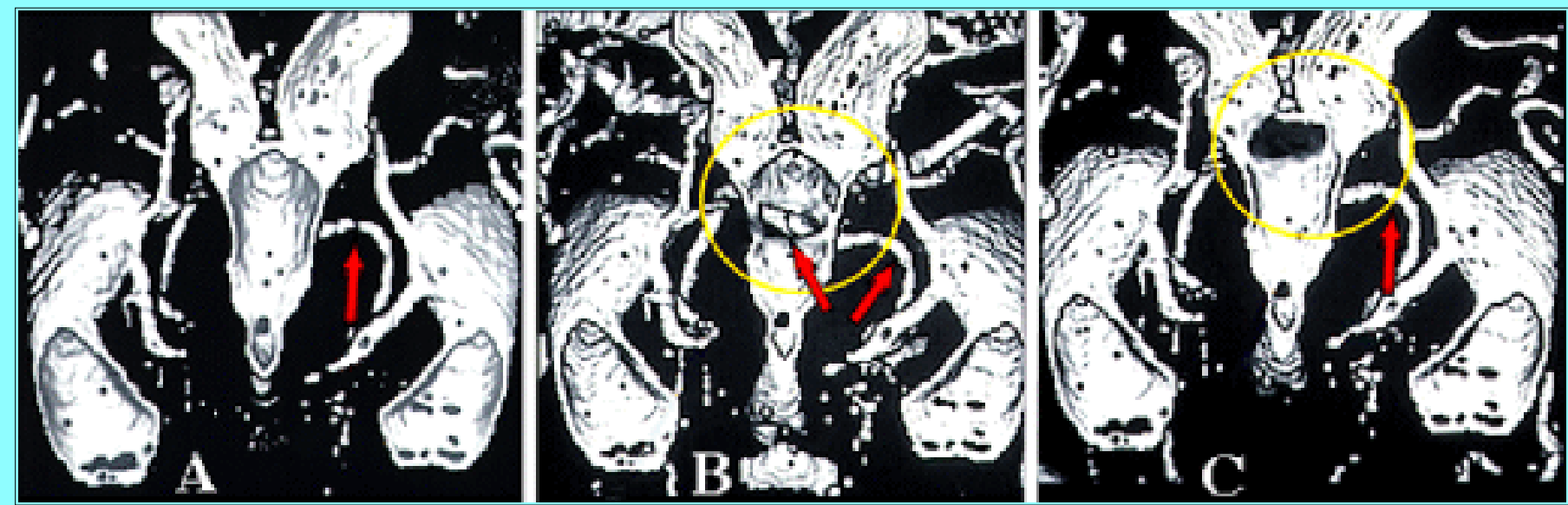

Fig. 10. Reconstructed combination of a virtual endoscopic view of the third ventricle and 3-D CT cerebral angiography documenting the anatomical relationship of the basilar artery to the anterior and posterior third ventricular floor. A: Pre-cut off view of third ventricle floor. B: Cut off view of the posterior part of the third ventricle (circle) floor. C: Cut off view of the anterior part of the third ventricle (circle) floor. Note that bilateral posterior cerebral arteries (P1) are located just underneath the posterior part of the third ventricle floor. Red arrows point to the posterior communicating artery. 


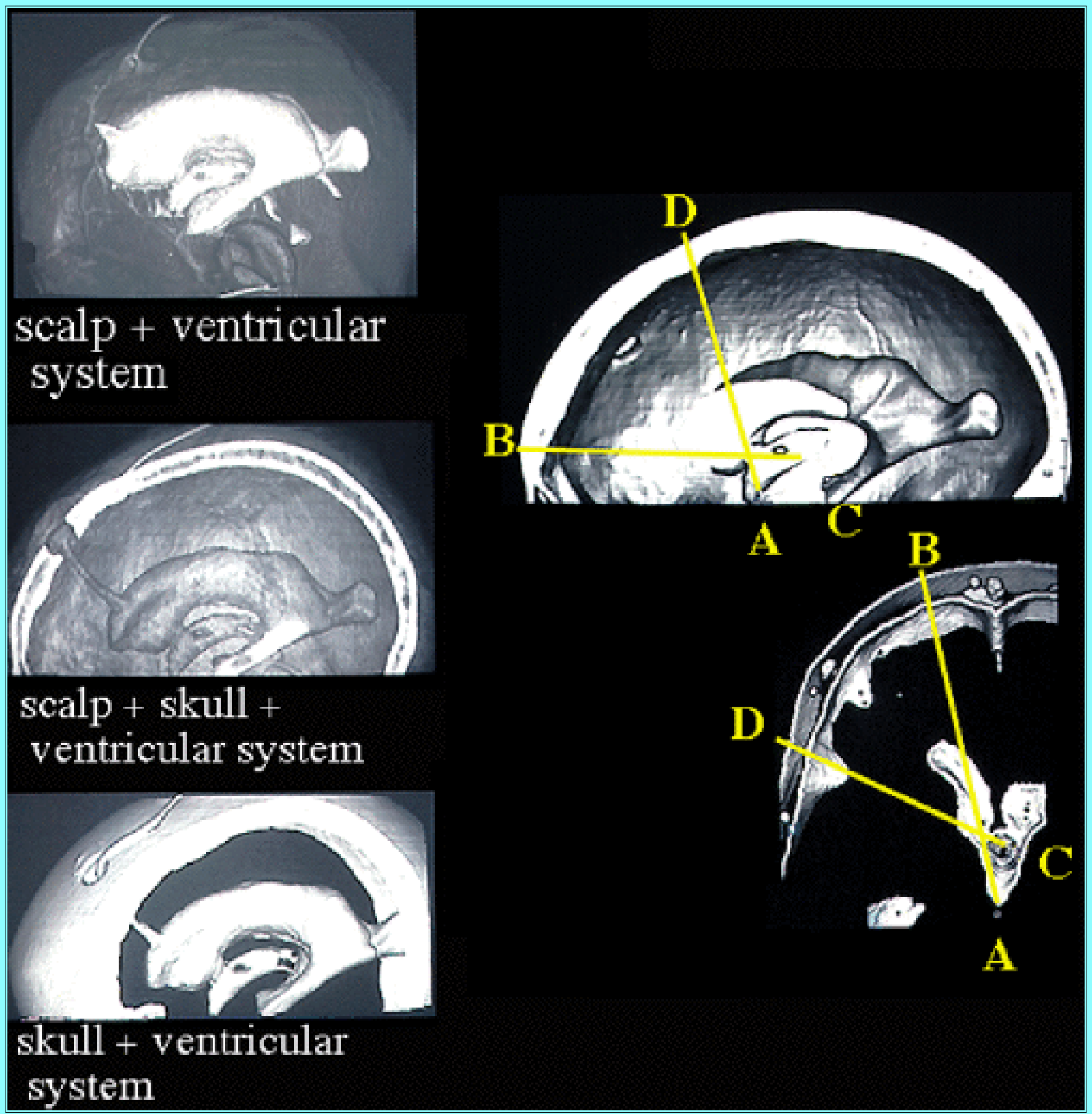

Fig. 11. Voxel transmission reconstruction of the skull and ventricular system allowing for preoperative evaluation of endoscopic entry and trajectory for maximal benefit during ventricular approach. Each target should have different burr hole placement on the skull during neuroendoscopic surgery. Placement of burr hole for neuroendoscope can be measured by combination of 3-D ventriculography and 3-D skull imaging. As noted in coordinate lines $\mathrm{B}$ to $\mathrm{C}$ and $\mathrm{D}$ to $\mathrm{A}$ in the sagittal section; $\mathrm{D}$ to $\mathrm{C}$ and $\mathrm{B}$ to $\mathrm{A}$ in the coronal view.

\section{TECHNICAL ISSUES TO BE SOLVED}

Several key issues regarding virtual endoscopic technique remain to be solved fully. These include resolution, thresholding and segmentation, visualization, and the real-time issue. 
Image resolution represents the fundamental impediment to improved virtual endoscopy. The thresholding that is used in the processing of these images may result in artifactual features. The use of virtual intraarterial endoscopy of the human aorta provides a good example of how techniques that rely on threshold algorithms may result in inaccurate renditions of the true anatomy. This may be based on varying degrees of pathological conditions that normally can be found.[9] Plaque formation or calcification of vessel lumens can result in variation in lumen size or even appearances of obstruction of the lumen, which may not be anatomically correct. These problems are based on the threshold level chosen by the operator. In addition, operator-mediated changes in threshold level can alter the appearance of small perforating vessels.

This effect can grossly impede any hopes of diagnostic results in the CNS, especially for endoscopically assisted aneurysm clip placement. Obviously improved vessel contrast in relation to the surrounding anatomy and smaller acquisition planes will allow one to better define small perforating vessels and to increase the diagnostic confidence in the virtual endoscope approach. In a recent study, reconstructions within the ventricle documented that normal thin wall elements appeared as artifacts and that the lamina terminalis and velum medullare superius were commonly missing. In addition, the quality of virtual reconstructions was poor in the subdural space, subarachnoid space, in demonstrating cystic cavities (namely for multicystic tumors or loculated hydrocephalus), and in thin membranes.[3] Although intravessel fly-throughs are possible with larger diameter vessels, current imaging technologies do not allow for obtaining image data sets that can be reconstructed into useful virtual fly-throughs of the circle of Willis.

The postprocessing of radiographic images also raises questions of anatomical fidelity. The overuse of sophisticated postprocessing techniques may obliterate small important features or may introduce artifacts that can be mistaken for a pathological or normal anatomical features.

Three-dimensional reconstructions of radiographic data are often difficult to interpret because of a phenomenon known as "aliasing;" those familiar with computer graphics know this phenomenon as "the jaggies." Because of the finite resolution of digital imaging, jagged edges and steps may appear where one knows a smooth suface should be present. Researchers in the early stages of computer graphics recognized this problem and found that a smoothing processes such as "antialiasing" could produce images that provided the illusion of higher resolution. Because of the relatively low-resolution nature of modern digitally based radiographic imaging, most such available commercial packages offer some degree of anti-aliasing. Anti-aliasing, however, must be used with the caveat that apparent resolution seen on a virtual image may reflect the true-image source resolution.

For example, application of antialiasing to smooth vessel walls can also result in the removal of important perforating vessels as well as smoothing of what may be appropriate and salient anatomy. Difficulties with multiprojection volume reconstructions are based on both the algorithms used to regenerate the 3-D image and the surgeon/operator's choice of voxel intensity for inclusion into the 3-D reconstruction. Based on these shortcomings, continuity of the reconstruction does not always correspond to the true anatomical detail, allowing for the potential exclusion or inclusion of important anatomical structures based on predetermined threshold values. In addition, overlapping or proximal structures may not be appropriately visualized and may, in fact, be smoothed or share the same homogenous surface if no specific density differences are recorded on the initial CT or MR imaging study. Studies to date have not allowed for the simultaneous imaging of parenchymal structures and vessels. The lack of vascular landmarks, including perforators, may make a detailed understanding of the anatomical correlates less 
feasible.

For all of these reasons virtual endoscopy does not compare with true endoscopic visualization to date. Most of these problems are directly related to interpolation algorithms, which are used to reconstruct surfaces in nonisotropic voxels. This results in artificial smoothing of surface structures and discrete stair-step artifacts that result from the pitch value of the helical CT scans being used. An additional problem is in the determination of threshold prior to the reconstruction, which results in a uniform display of all pixels based on the defined density level. This can interfere with the surgeon's ability to differentiate structures, whether anatomically normal or abnormal, that are adherent or adjacent to other anatomical structures. One way to deal with this problem is to enhance the spatial resolution by using smaller fields of view or smaller CT slices. Obviously the use of smaller CT slices would potentially require higher doses of radiation and might not be appropriate.

A work-around solution to the problems regarding resolution and postprocessing may lie in multimodality image fusion. In this method, a superimposition of different 3-D reconstructions within the same stereotactic space is obtained. For example, CT, MR, MR angiography, functional MR, and positron emission tomography data may be merged into a single stereotactic space to allow delineation of anatomical features not viewable with a single modality alone.

\section{Visualization Probems}

Other problems are related to resolution. Intraarterial digital subtraction angiography continues to be superior to 3-D reconstructions using either CT angiography or MR angiography data sets. Unfortunately reconstruction of intraarterial digital subtraction angiograms into 3-D volumes has been, for the most part, unsuccessful to date. Newer technological developments continue to improve the resolution following MR angiography. These include 2- or 3-D time-of-flight imaging and phase-contrast imaging. These modalities can also allow for better resolution of intracranial vascular lesions.[5,9,33]

Unfortunately, despite these improvements, MR angiography and CT angiography still cannot identify small perforating vessels, which remain crucial to the management of cerebral aneurysms. In addition, irregularities resulting from calcified aneurysmal domes or necks as well as artifact from high-velocity flow within the parent vessels or aneurysmal dome remain problematic. On the other hand, 3-D reconstructions can provide a better understanding of the projection of the aneurysm neck and fundus in relation to the parent vessels.[31] These diagnostic studies allow for useful information to plan the approach for clip ligation of these lesions.

\section{Real-Time Problem}

A final problem with virtual endoscopic reconstructions is that they are based on preoperative images and, thus, in no way can take into account shift as a result of surgical debulking, the use of hyperventilation osmotic agents, or the drainage of cerebrospinal fluid from the ventricular system or cisterns.

Unfortunately the derivation of such data from the baseline CT or MR imaging cross-sectional data can be time consuming (the average amount of computer time required for data preparation can range from 6 to 10 hours). This is based on the difficulty of software algorithms in defining specific tissue planes in scenarios in which tissue differences may not be perceived adequately based on CT or MR images. Such extended postprocessing times may limit the application of intraoperative imaging techniques for the near future. 


\section{DISCUSSION}

There is no doubt that virtual endoscopy remains a nascent field, largely due to technical issues. These issues will, to a large extent, be resolved in the next few years, hopefully one day becoming nonexistent. Significant work remains to be performed in the realm of direct clinical application of these methods. The accuracy and reproducibility of 3-D rendering techniques in the visualization of both normal and abnormal anatomy needs to be established.[14,45,46] Clinically, an additional potential issue is the cost effectiveness of virtual endoscopy and the issue of whom should be completing these studies. The issue of professional sovereignty regarding this technology will obviously become a question of interest between radiologists, neurologists, and neurosurgeons in the near future.

\section{Regulation and Reimbursement}

The questions of whether virtual neuroendoscopy is eventually approved by the United States Food and Drug Administration and eventually given its own current procedural terminology code by the American Medical Association have yet to be answered. The setting of relative value units and fees by the Health Care Financing Administration will also need to be defined. Obviously a low cost attached to these procedures is important if they are ever to be considered as initial diagnostic modalities. Given customary billing, in which independent CT scans of the head and neck examine the same anatomical regions, there may be no cost advantage to performing virtual endoscopy in the brain. Although current procedural terminology billing codes do exist for the performance of 3-D reconstructions with spiral CT data (code 76374), the current reimbursement is not adequate to perpetuate the use of these technologies in the private sector. It is likely, therefore, that these methods will continue only within the realm of research or education.

\section{CONCLUSIONS}

To date, the only potential way to compensate for errors that exist in the algorithms and reconstructions of 3-D endoscopic images is based on the surgeon's understanding of the clinical state of the patient and prior experience with the anatomy in the region of question. One must always be cognizant of the technical limitations of these studies despite the quality of the reconstruction. Comparative clinical studies in which the advantages of virtual-based endoscopy is evaluated in large cadaver or patient series have yet to be performed. In addition, continuous comparison with standard axial, coronal and sagittal MR imaging or CT scanning cuts can further allow for the surgeon to verify position, continuity, and accuracy of the anatomy that is being evaluated. We recommend that processing be performed by a team including neurosurgeons and neuroradiologists.[36] Obviously, although virtual endoscopic images at this time are in no way a replacement for actual endoscopic diagnostic techniques in the brain or elsewhere, they do provide significant benefits not only in education but in simultaneous display during endoscopic and endoscopically assisted open procedures.

\section{References}

1. Apuzzo MLJ: The Richard C. Schneider Lecture. New dimensions of neurosurgery in the realm of high technology: possibilities, practicalities, realities. Neurosurgery 38:625-639, 1996

2. Auer DP, Auer LM: Virtual endoscopy: a new tool for teaching and training in neuro-imaging. Int J Neuroradiol 4:3-14, 1998 
3. Auer LM, Auer DP: Virtual endoscopy for planning and simulation of minimally invasive neurosurgery. Neurosurgery 43:529-548, 1998

4. Blezek DJ, Robb RA: Evaluating virtual endoscopy for clinical use. J Digit Imaging 10 (Suppl 1):51-55, 1997

5. Brown DG, Riederer SJ, Jack CR, et al: MR angiography with oblique gradient-recalled echo technique. Radiology 176:461-466, 1990

6. Buthiau D, Antoine E, Piette JC, et al: Virtual tracheo-bronchial endoscopy: educational and diagnostic value. Surg Radiol Anat 18:125-131, 1996

7. Clark MC, Hall LO, Goldgof DB, et al: Automatic tumor segmentation using knowledge-based techniques. IEEE Trans Med Imaging 17:187-201, 1998

8. Clarke LP, Velthuizen RP, Camacho MA, et al: MRI segmentation: methods and applications. Magn Reson Imaging 13:343-368, 1995

9. Davis CP, Ladd ME, Romanowski BJ, et al: Human aorta: preliminary results with virtual endoscopy based on three-dimensional MR imaging data sets. Radiology 199:37-40, 1996

10. De Nicola M, Salvolini L, Salvolini U: Virtual endoscopy of nasal cavity and paranasal sinuses. Eur J Radiol 24:175-180, 1997

11. Fellner F, Blank M, Fellner C, et al: Virtual cisternoscopy of intracranial vessels: a novel visualization technique using virtual reality. Magn Reson Imaging 16:1013-1022, 1998

12. Fried MP, Hsu L, Jolesz FA: Interactive magnetic resonance imaging-guided biopsy in the head and neck: initial patient experience. Laryngoscope 108:488-493, 1998

13. Fried MP, Hsu L, Topulos GP, et al: Image-guided surgery in a new magnetic resonance suite: preclinical considerations. Laryngoscope 106:411-417, 1996

14. Gilani S, Norbash AM, Ringl H, et al: Virtual endoscopy of the paranasal sinuses using perspective volume rendered helical sinus computed tomography. Laryngoscope 107:25-29, 1997

15. Glassner AS: An Introduction to Ray Tracing. New York: Academic Press, 1989

16. Harpold T, Apuzzo M, McComb J, et al: Virtual-assisted microneurosurgery. Comput Assist Radiol Surg 11:904-908, 1997

17. Harris GJ, Barta PE, Peng LW, et al: MR volume segmentation of gray matter and white matter using manual thresholding: dependence on image brightness. AJNR 15:225-230, 1994

18. Hu X, Alperin N, Levin DN, et al: Visualization of MR angiographic data with segmentation and volume-rendering techniques. J Magn Reson Imaging 1:539-546, 1991

19. Jolesz FA: Interventional and intraoperative MRI: a general overview of the field. J Magn Reson Imaging 8:3-7, 1998

20. Jolesz FA, Lorensen WE, Shinmoto H, et al: Interactive virtual endoscopy. AJR 169:1229-1235, 1997 
21. Kalender W, Seissler W, Klotz E, et al: Spiral volumetric CT with single breath hold technique, continuous transport, and continuous scanner rotation. Radiology 76:180-183, 1990

22. Kay CL, Evangelou HA: A review of the technical and clinical aspects of virtual endoscopy. Endoscopy 28:768-75, 1996

23. Kochanek D, Bartels R: Interpolating splines with local tension, continuity, and bias control. Comput Graph 18:33-41, 1984

24. Koechner D, Petropoulos H, Eaton RP, et al: Segmentation of small structures in MR images: semiautomated tissue hydration measurement. J Magn Reson Imaging 5:347-51, 1995

25. Lee DH: Three-dimensional imaging of the stomach by spiral CT. J Comput Assist Tomogr 22:52-58, 1998

26. Lengyel J, Reichert M, Conald B, et al: Real-time robot motion planning using rasterizing. Comput Graph 24:327-335, 1990

27. Levy ML, Chen C, Harpold TL, et al: Technical advances in neurosurgery: computer assisted imaging and surgery. Adv Clin Neurosci 7:1-16, 1997

28. Levy ML, Chen JC, Moffitt K, et al: Stereoscopic head-mounted display incorporated into microsurgical procedures: technical note. Neurosurgery 43:392-396, 1998

29. Levy ML, Weinberg RA, Valencia P, et al: Information flow and imaging technology in the operating room, in Salcman M (ed): Current Techniques in Neurosurgery, ed 2. Philadelphia: Current Medicine, 1996, pp 4-14

30. Lin JS, Cheng KS, Mao CW: Segmentation of multispectral magnetic resonance image using penalized fuzzy competitive learning network. Comput Biomed Res 29:314-326, 1996

31. Marro B, Galanaud D, Valery CA, et al: Intracranial aneurysm: inner view and neck identification with CT angiography virtual endoscopy. J Comput Assist Tomogr 21:587-589, 1997

32. McGregor JM: Enhancing neurosurgical endoscopy with the use of virtual reality' headgear. Minim Invasive Neurosurg 40:47-49, 1997

33. Mehta MP, Petereit D, Turski P, et al: Magnetic resonance angiography: a three-dimensional database for assessing arteriovenous malformations. Technical note. J Neurosurg 79:289-293, 1993

34. Melgar MA, Zamrano L, Jiang Z, et al: Three-dimensional magnetic resonance angiography in the planning of aneurysm surgery. Comput Aid Surg 2:11-23, 1997

35. Phillips WE II, Velthuizen RP, Phuphanich S, et al: Application of fuzzy c-means segmentation technique for tissue differentiation in MR images of a hemorrhagic glioblastoma multiforme. Magn Reson Imaging 13:277-290, 1995

36. Robb RA: Computer aided surgery planning and rehearsal at the Mayo Clinic. J Comput Aid Surg 1:36-37, 1995

37. Rodenwaldt J, Kopka L, Roedel R, et al: 3D virtual endoscopy of the upper airway: optimization of 
the scan parameters in a cadaver phantom and clinical assessment. J Comput Assist Tomogr 21:405-411, 1997

38. Rogalla P, Nischwitz A, Gottschalk S, et al: Virtual endoscopy of the nose and paranasal sinuses. Eur Radiol 8:946-950, 1998

39. Rubin GD, Beaulieu C, Argiro V, et al: Perspective volume rendering of CT and MR images: applications for endoscopic imaging. Radiology 199:321-330, 1996

40. Rubin GD, Dake MD, Napel S, et al: Spiral CT of renal aretery stenosis: comparison of three dimensional rendering techniques. Radiology 190:181-189, 1994

41. Saeed N: Magnetic resonance image segmentation using pattern recognition, and applied to image registration and quantitation. NMR Biomed 11:157-167, 1998

42. Satava RM: Virtual endoscopy: diagnosis using 3-D visualization and virtual representation. Surg Endosc 10:173-174, 1996

43. Shigematsu Y, Korogi Y, Hirai T, et al: Virtual MRI endoscopy of the intracranial cerebrospinal fluid spaces. Neuroradiology 40:644-650, 1998

44. Vining DJ: Virtual colonoscopy. Gastrointest Endosc Clin North Am 7:285-291, 1997

45. Vining DJ: Virtual endoscopy: is it reality? Radiology 200:30-31, 1996

46. Vining DJ, Liu K, Choplin RH, et al: Virtual bronchoscopy. Relationships of virtual reality endobronchial simulations to actual bronchoscopic findings. Chest 109:549-553, 1996

47. Warfield S, Dengler J, Zaers J, et al: Automatic identification of gray matter structures from MRI to improve the segmentation of white matter lesions. J Image Guid Surg 1:326-338, 1995

48. Wood BJ, O'Malley ME, Hahn PF, et al: Virtual endoscopy of the gastrointestinal system outside the colon. AJR 171:1367-1372, 1998

49. Zamorano L, Jiang Z, Kadi AM: Computer-assisted neurosurgery system: Wayne State University hardware and software configuration. Comput Med Imaging Graph 18:257-271, 1994

Manuscript received February 15, 1999.

Accepted in final form March 15, 1999.

Address reprint requests to: Michael L. Levy, M.D., 1300 North Vermont Avenue, Suite 906, Los Angeles, California 90027. 